Horizons philosophiques

\title{
Les variétés des hybrides dans les récits de la pensée
}

\section{Daniel Canty}

Volume 5, numéro 2, printemps 1995

Le philosophe et l'état

URI : https://id.erudit.org/iderudit/800985ar

DOI : https://doi.org/10.7202/800985ar

Aller au sommaire du numéro

\section{Éditeur(s)}

Collège Édouard-Montpetit

\section{ISSN}

1181-9227 (imprimé)

1920-2954 (numérique)

Découvrir la revue

Citer cet article

Canty, D. (1995). Les variétés des hybrides dans les récits de la pensée. Horizons philosophiques, 5(2), 132-141. https://doi.org/10.7202/800985ar d'utilisation que vous pouvez consulter en ligne.

https://apropos.erudit.org/fr/usagers/politique-dutilisation/ 


\section{LES VARIÉTÉS DES HYBRIDES DANS LES RÉCITS DE LA PENSÉE*}

You know that ad, that ad for the phone company, there's a mother and her little daughter and they're sitting around and the phone rings. The mother picks it up and talks for a minute. Then she holds the receiver out to the daughter and says, «Talk to grandma! Here's grandma!" And she's holding a piece of plastic. So, of course, the question is : Is the phone alive? What's alive and what doesn't have life?

Laurie Anderson, Stories from the Nerve Bible

Des robots arachnides descendentà la place des spécialistes humains dans les cratères des volcans. Des camions autonomes convoient du minérai extrait par des pelles automatisées jusqu'aux sites de traitement. De Three Mile Island à Tchernobyl, des unités mobiles quadrillent les périmètres ravagés par la radioactivité. Des techniciens imaginent des machines pour dégorger les égoûts des villes et nettoyer jusqu'aux panneaux les plus élevés des gratte-ciel'. Les ingénieurs de la NASA rêvent d'envoyer une nuée de robots parcourir les ravins sablonneux de Mars et les glaces d'Europe ${ }^{2}$. Des téléscopes automates balaient en orbite l'univers entier de leur regard radio, dilatant notre œil si grand qu'on espére un jour apercevoir les confins du monde, d'où les plus vieilles étoiles content le récit de nos origines ${ }^{3}$. Partout où l'homme en allant risque la mort dans le miasme ou le vide, et où le réel en ses bords défie

- L'auteur a bénéficié d'une bourse du fonds FCAR pendant la rédaction de ce texte.

1. Sur les variétés des robots, voir l'entrevue accordée par le professeur Red Whittaker (concepteur de "Dante" et "Ambler") de l'université Carnegie Mellon à Omni , janvier 1994, p. 73-77 et p. 95-101.

2. Pierre Léna décrit ces projets dans L'espace pourl'homme,Paris, Flammarion, coll. Dominos, 1993, p. 27-40.

3. Alan Lightman, l'auteur d'une belle "vie imaginaire» d'Einstein, Einstein's Dreams, New York, Warner Books, 1993, décline dans Time for the Stars, New York, Warner Books, 1992, p. 109-111, la liste des appareils de notre regard astronomique. 
les sens, il délègue les non-humains. La multiplication de leur nombre mime celle des espaces qui s'ouvrent alors à notre intervention, à notre connaissance et à notre curiosité. À chaque fois qu'un robot lâché dans le monde repousse les limites de l'empirie, il trace en même temps une nouvelle frontière du concevable.

Les machines n'arpentent pas seulement des territoires matériels : elles courent plutôt surl'entière étendue du pensable. Dans les laboratoires universitaires, à Carnegie-Mellon, à Caltech, Cornell ou au MIT, ou dans des sous-sols de banlieue, des programmeurs donnent naissance à d'étranges hybrides, des êtres de software, habitants d'un troisième monde (Popper) situé à égale distance des sphères chantantes de la raison pure et des dépotoirs de l'empirie. Depuis que John Von Neumann a formulé sa théorie des automates cellulaires, depuis aussi que John Horton Conway a voulu y deviner les règles d'un jeu qu'il nommera du nom de la vie - The Game of Life - et que Fred Cohen s'est vu suspendre les vivres pour avoir conçu le premier des "virus informatiques", des formes nouvelles courent surles mailles du réseau, qu'un entier champ de recherche, celui de la "vie artificielle» (artificial life), consacre son temps à étudier. Des "ontologiciens" génèrent sur les ordinateurs à partir d' «algorithmes génétiques" des scénarios évolutifs, qui produisent dans des écosystèmes de fabrication des êtres inédits ${ }^{4}$. D'aucuns disent qu'ils bénéficient d'une demi-vie, qui n'a rien à voir avec celle des particules, mais qui permet de les situer sur l'échelle des êtres à mi-chemin entre le sujet et l'objet, et de les compter au nombre de ceux que Michel Serres a choisi de désigner quasi-objets, et dont Bruno Latour après lui a approfondi l'étude 5 .

Les nouvelles variétés des hybrides mènent dans leurs univers intercalaires des vies qui sont riches d'imaginaire, et qui renouvellent l'ancestrale pratique de la gedankenexperiment,

4. Stephen Levy dresse l'historique de la "vie artificielle" dans son ouvrage, Artificial Life, New York, Vintage, 1992.

5. Dans Nous n'avons jamais été modernes (Paris, La Découverte, 1991). 
qui a maintenant lieu dans un quasi-monde dont on dirait qu'il bénéficie, par rapport au domaine de la pure intellection, d'un surplus de réalité. La littérature déjà connaissait cet espace intermédiaire, où les personnages des "récits de la pensée» 6 élisent depuis toujours demeure. Dans le lignage des hybrides, sans doute ces produits des technologies du verbe sont-ils, avant les machines cognitives et les êtres bio-computationnels, les premiers en date. Ce ne sont pas pour autant les plus primitifs : les ingénieurs cogniticiens ne font bien souvent que reprendre dans le travail de la silice et du micro-processeur, des rêves que la littérature ou la philosophie depuis longtemps déjà cultivaient.

Je voudrais retracer la parenté de Teste ${ }^{7}$, Palomar $^{8}$, Glot $^{9}$ et Songe ${ }^{10}$ avecle virus, la prothèse oul'automate, qui fournissent ses emblèmes au règne des hybrides. On sait maintenant du virus qu'il mène une existence de mort-vivant : aussi inerte que la pierre lorsqu'il flotte dans l'air, il rompt sa corolle et reprend vie lorsqu'il pénètre l'animal pour l'infecter. On retrouve la même interpénétration de l'humain et du non-humain, du vivant et de l'inerte, dans un appareil comme la jambe du Capitaine Ahab ${ }^{11}$, qui représente une expression minimale de la logique de la prothèse, dont la fonction est toujours de prolonger le rayon d'action des sens ou de l'intellect à travers l'artifice. L'automate, lorsqu'il se fait androïde, porte à son extrême extension la logique de la prothèse, en substituant à l'ensemble de l'individu un mécanisme qui lui ressemble et peut dans l'idéal s'acquitter aussi bien que l'homme de ses fonctions cognitives.

Jean-Claude Beaune a répertorié, dans la somme d'automatisme philosophique qu'est L'automate et ses mobiles, les versions de l'automate ${ }^{12}$. Intériorisant le principe de son propre

6. C'était là le titre du séminaire animé par Pierre Ouelletà l'UQAM en1994. M. Ouellet prépare actuellement un ouvrage à ce propos.

7. Valéry, Paul, Monsieur Teste, Paris, Gallimard, L'imaginaire, 1946.

8. Calvino, Italo, Palomar, Paris, Seuil, Points, 1985 (1983).

9. Roegiers, Patrick, L'horloge universelle, Paris, Seuil, 1992.

10. Pinget, Robert, Monsieur Songe, Paris, Minuit, 1985.

11. Melville, Herman, Moby Dick, London, Everyman's Library, 1992 (1907). 
mouvement, l'automate bénéficie (comme son nom l'indique) d'une autonomie qui lui permet de maintenir le leurre de son individualité, en même temps qu'elle éveille dans les consciences qui se délectent de son spectacle le soupçon de leur appartenance à un mécanisme universel. La performance de l'automate met la conscience à l'épreuve de ses règles, dont elle lui force à conjecturer l'existence, lui lançant par le fait même un défi à en dépasser l'horizon pour accéder à la connaissance de son irréductible singularité ${ }^{13}$. À travers l'automate, les mécanismes de la conscience engendrent la conscience du mécanisme, qui représente la condition paradoxale et nécessaire à son propre dépassement ${ }^{14}$.

Les personnages des récits de la pensée éveillent chez leur lecteur le même soupçon. La filiation de ce "monstre d'attention" qu'est Monsieur Teste à l'automate est indéniable. Son nom la racine latine de "tête" - rappelle la tête parlante et omnisciente sculptée dans l'airain par Roger Bacon. Test est également l'héritier de Da Vinci et Descartes, qui rêvaient l'un comme l'autre de réduire la part animale de l'homme à une machine ou à un assemblage de celles-ci. En fait, peut-être est-ce Descartes qui donne dans le Discours de la méthode la première esquisse de Teste, dont Valéry revisera le bleu à la lumière de la topologie de Poincaré.

Descartes voulait dissocier le corps asservi à ses impératifs machiniques d'un esprit conscient de sa liberté. Sa démarche, plutôt que de marquer le triomphe du dualisme, confine au paradoxe en engendrant, au-dessus de l'automate corporel, une machine surrationnelle, dont Teste représente sans aucun doute une des incarnations les plus achevées. Il faut se demander si le rationalisme cartésien n'est pas toujours déjà un surration-

12. Beaune, Jean-Claude, L'automate et ses mobiles, Paris, Flammarion, 1980.

13. Je m'inspire ici des travaux de Paul Zumthor sur la notion de performance, voir Performance, réception, lecture, Candiac, Balzac, 1990.

14. Cet argument a déjà été développé par Jean-Pierre Chopin à la lumière de l'œuvre de Valéry dans "La grande leçon de Valéry ou l'intelligence artificielle comme acomédie de l'espritw», Milieux, \#30, 1987, p. 20-25). 
nalisme. En effet, si Descartes fournit au sujet, à travers la méthode analytique du doute systématique, un moyen de «tuer la marionnette", c'est à travers le "crible machinal» ${ }^{15}$ en quoi consiste l'imitation du Dieu, contenant en toutes situations sa volonté dans les limites de son entendement ${ }^{16}$ (entendre : de ce qui lui est connu). II semblerait donc que la rigueur procédurale de la méthode n'ait rien à envier à celle des machines cognitives et que le paradoxe de l'entreprise cartésienne tienne à ce que l'émancipation de l'esprit par rapport à la machine corporelle et à ses fonctionnements inconscients passe par l'application d'une volonté consciente - sinon d'une attention monstrueuse -à se conformer au comportement d'un automate cognitif dont la raison divine fournit le modéle. La conscience individuelle se transforme alors en un mécanisme auto-régulé selon les préceptes d'une rationalité suprême qui se subordonne son expérience singulière, et qui mérite par là l'appellation d'automate surrationnel. Si un individu parvenait à s'éveiller à la surrationnalité, il pourrait répondre comme Testeà quil'interroge surce qu'il pense, que "Personne ne médite»" 17 . Qui se conforme à la méthode doit nécessairement résorber sa singularité dans l'abstraction universalisante de la Rationalité suprême, et devenir "le démon même de la possibilité» ${ }^{18}$, qui joue de l'entière étendue du concevable comme s'il s'agissait de son être.

Teste, pas plus qu'il ne distingue les autres du continu («ll parlait, et on se sentait dans son idée, confondu avec les choses : on se sentait reculé, mêlé aux maisons, aux grandeurs de l'espace, au coloris remué de la rue, aux coins ${ }^{19}$ "), ne s'en détache. Le narrateur peut dire qu'il "était l'être absorbé dans sa variation, celui qui devient son système ${ }^{20}$ ", dans laquelle phrase il faudrait lire l'être avec un «ÊE majuscule. Teste est

15. Valéry, P., Monsieur Teste, p. 19.

16. L'essentiel de la méthode cartésienne est présenté dans les Méditations métaphysiques, Paris, Le livre de poche et le Discours de la méthode, Paris, Le livre de poche.

17. Valéry, P., Monsieur Teste, p. 27.

18. Ibid., p. 11.

19. Ibid., p. 21.

20. Ibid., p. 20-21. 
l'homme qui refuse "de se considérer comme autre chose [qu'une chose] ${ }^{21}$ ", l'hybride qui à l'image de l'automate cognitif parmi les choses les pense, usant de l'intelligence comme d'un artifice à travers lequel la pensée pourrait retourner au domaine du concevable qui l'avait vu en sortir, sous l'emprise du monde dont elle se distinguait en le posant devant sa subjectivité en objet. Teste ressemble en cela à Palomar, qui se demande "comment faire pour regarder quelque chose en mettant de côté le moi?" et "devenir un morceau du monde en train de regarder un autre morceau du monde ${ }^{22}$ ".

Teste ressemble également à Glotz, qui apprend la voix en modulant le flot continu des sonorités, plutôt qu'en copiant les pratiques sociales qui nous apprennent à hachurer les phonèmes. De même que Glotz appréhende la vocalité comme la manipulation d'un tissu sonore, Teste joue de l'être comme d'une surface topologique en transformation continue. De la même manière que Teste joue de lui-même comme d'un instrument du possible, Glotz est tout entier l'instrument de sa voix. Par sa chute finale en lui-même, Glotz rejoint le tissu même del'espace-temps, dont Teste n'avait de cesse d'éprouver la malléabilité.

Songe également, lorsqu'il se plaît à varier du possible sur le mode à proprement parler algorithmique du branchement conditionnel (structure de type si/alors) ressemble à une machine à compiler du possible, qui ramène au réel tout ce que l'événement, à travers le choix qu'il implique, exclut d'advenir. Témoin ce dessert de Songe, qui prend les allures d'une arborescence décisionnelle complexe. Il suggère que l'anecdote même la plus insignifiante, recèle une complexité cachée que chaque instant demande l'application d'une inflexible rigueur procédurale :

La bonne revient, débarrasse les reliefs, pose le fromage devant son maître qui dit soit est-il bien fait si c'est du camembert, soit est-il bien gras si c'est du gruyère, soit estil de Bresse si c'est du bleu. La bonne répond oui, dépose

21. Ibid., p. 18.

22. Calvino, Palomar, p. 112. 
les fruits et se retire. II mange alors son fromage dans la même attitude pensive que précédemment, puis un fruit. $\mathrm{Si}$ c'est une pomme il la pèle avec son couteau en la faisant tourner de la main gauche sous la lame de telle sorte qu'un seul ruban de pelure tombe dans l'assiette en fin d'opération. II la coupe ensuite en quatre quartiers selon l'axe vertical et de chacun extrait le morceau de trognon comme il dit qui contient les pépins.

Si c'est une orange, il trace sur l'écorce avec son couteau un premier sillon circulaire en partant du haut et en faisant tourner le fruit de haut en bas, puis un second sillon à angle droit de telle sorte que l'écorce ainsi divisée en quatre segments se détache aisément à l'aide des doigts. II mange alors l'orange côte par côte en crachant les pépins dans sa main droite qui les laisse tomber dans l'assiette.

Si c'est une banane [etc. $]^{23}$

Une fois constatée cette parenté machinique des personnages des récits de la pensée, on peut revenir à Descartes pour aborder leurs automatismes cognitifs sous un autre angle. On sait que Descartes s'inspirait, dans la formulation de ses préceptes épistémiques, de ses travaux en géométrie analytique, et qu'il envisage le procès de la méthode comme celui de la découverte de vérités analytiques, qu'il est impossible de penser autrement sans contradiction et que le seul travail de la reconstruction rationnelle devrait permettre de découvrir. L'esprit méthodique, se conformantà l'idéal nomologique de la science, procède dans la vie comme s'il s'agissait d'un calcul. Une telle perspective fait de Descartes le précurseur des théories computationnelles de l'esprit propres aux sciences cognitives, selon lesquelles l'activité de l'esprit est réductible à un calcul s'effectuant sur un support matériel, à ceci près que Descartes, au contraire des cogniticiens, fait de la res cogitans, dénuée de toute matérialité, le site des processus de la conscience. Le computo, comme disait Edgar Morin, se substitue aujourd'hui au cogito ${ }^{24}$ en y ajoutant sa dimension matérielle.

23. Pinget, Monsieur Songe, p. 19.

24. voir La méthode 3. La connaissance de la connaissance, Paris, Seuil, Points essais, 1986. 
Dans les récits de la pensée, on dirait que l'activité de calcul de la géométrie analytique est remplacée par l'analyse topologique d'un Poincaré, l'automate surrationnel qu'est Teste computant sans cesse sur du continu comme s'il s'agissait de l'unique et indivisible substance du monde : il «opérait tout ce qui lui était proposé $e^{25}$ » pour mener à bien «quelque expérience créée aux confins de toutes les sciences ${ }^{26}$ " et consistant à chercher "les limites et le mécanisme» "de ce qu'on pourrait nommer la plasticité humaine ${ }^{27}$ ». L'insistance de Songe à décliner les généalogies du possible, que Palomar aussi parfois adopte, participe d'une même obsession à ramener au réel tout ce que l'événement en exclut, ajoutant à la perception empirique la richesse de mille mondes possibles. Les travaux en intelligence ou en vie artificielle montrent que les ingénieurs aussi ont fini par croire devant leurs machines, comme la narrateur de la "soirée avec $M$. Teste" devànt son hôte aux allures d'automate ${ }^{28}$, qu'il est possible d'arriver "à découvrir des lois de l'esprit que nous ignorons ${ }^{29}$ " et de ramener au réel grâce aux variétés des hybrides ce qui pouvait nous en manquer.

Daniel Canty Études littéraires, UQAM

25. Valéry, P., Monsieur Teste, p. 23.

26. Ibid., p. 27.

27. Ibid., p. 20.

28. Teste a la parole sourde et le regard aveugle des choses à demi-animées que Glotz acquerra dans son involution : "Sa parole était extraordinairment rapide, et sa voix sourde. Tout s'effaçait en lui, les yeux, les mains". Son pas est "d'une régularité qui étonnait" et ses mouvements sont entièrement dictés par son attention surrationnelle : "Quand il parlait, il ne levait jamais un bras ni un doigt [...] II ne souriait pas, ne disait ni bonjour ni bonsoir", p. 19. II semblerait bien que le meurtre de la marionnette amène la naissance de l'automate.

29. Ibid., p. 19. 


\section{Bibliographie}

Beaune, Jean-Claude, L'automate et ses mobiles, Paris, Flammarion, 1980.

Calvino, Italo, Palomar, Paris, Seuil, Points, 1985, 1983.

Chopin, Jean-Pierre, "La grande leçon de Valéry ou l'intelligence artificielle comme "comédie de l'esprit»», Milieux. \#30, 1987, p. 20-25.

Descartes, René, Discours de la méthode, Paris, Le livre de poche.

Descartes, René, Méditations métaphysiques, Paris, Lelivre de poche.

Latour, Bruno, Nous n'avons jamais été modernes, Paris, La Découverte, 1991.

Léna, Pierre, L'espace pour l'homme, Paris, Flammarion, coll. Dominos, 1993.

Levy, Stephen, Artificial Life, New York, Vintage, 1992.

Lightman, Alan, Einstein's Dreams, New York Warner Books, 1993.

Lightman, Alan, Time for the Stars, New York, Warner Books, 1992.

Melville, Herman, Moby Dick, London, Everyman's Library, 1992 (1907).

Moravec, Hans, Mind Children, Cambridge, Harvard University Press, 1988

Morin, Edgar, La méthode 3. La connaissance de la connaissance, Paris, Seuil, Points, 1986.

Pinget, Robert, Monsieur Songe, Paris, Minuit, 1985.

Roegiers, Patrick, L'horloge universelle, Paris, Seuil, 1992.

Valéry, Paul, Monsieur Teste, Paris, Gallimard, L'imaginaire, 1946. 
Whittaker, Red, «Interview», Omni, Janvier 1994.

Zumthor, Paul, Performance, réception, lecture, Candiac, Balzac, 1990. 